\title{
What is the fate of repeat biopsies after diagnosis of high grade prostatic intraepithelial neoplasia and atypical small acinar proliferation?
}

\author{
Yakup Bostanci $^{\text {a* }}$ Ender Ozden ${ }^{\mathrm{a}}$, Yarkin Kamil Yakupoglu ${ }^{\mathrm{a}}$, Serdar Savasci ${ }^{\mathrm{b}}$, Ali Faik Yilmaz ${ }^{\mathrm{a}}$, Saban Sarikaya $^{\mathrm{a}}$ \\ ${ }^{a}$ Department of Urology, Faculty of Medicine, Ondokuz Mayls University, Samsun, Turkey \\ ${ }^{b}$ Department of Urology, Bayburt State Hospital, Bayburt, Turkey
}

\section{ARTICLE INFO}

\section{Article History}

$\begin{array}{ll}\text { Received } & 13 / 06 / 2013 \\ \text { Accepted } & 15 / 08 / 2013\end{array}$

\section{* Correspondence to:}

Yakup Bostanci

Department of Urology,

Faculty of Medicine,

Ondokuz Mayıs University,

Samsun, Turkey

e-mail: dryakupbostan@yahoo.com

\section{Keywords:}

Atypical small acinar proliferation

Biopsy

Prostate carcinoma

Prostatic intraepithelial neoplasia

Prostate specific antigen

\section{ABSTRACT}

In this study, we reviewed the outcomes of patients undergoing repeat biopsies, following initial diagnosis of high grade prostatic intraepithelial neoplasia (HGPIN) or atypical small acinar proliferation (ASAP) and compared the pathological results to second biopsies due to increased PSA. We retrospectively assessed transrectal ultrasound guided prostate biopsy (TRUSBP) database at our institution between January 2003 and March 2011. Nonparametric tests and binary logistic regression analysis was performed. Among the 1451 TRUSBP taken, $30.4 \%, 6.4 \%, 4.7 \%$ were diagnosed as prostate carcinoma (PCa), HGPIN, ASAP, respectively. Among patients with HGPIN and ASAP, 68 patients and 48 patients with subsequent biopsies were selected. We also selected 128 patients with diagnosis of benign prostatic tissue (BPT) and subsequent biopsies due to increased PSA. After second biopsy, HGPIN and PCa reported in $29.4 \%$ and $20.6 \%$, respectively in HGPIN group; ASAP and PCa was reported in $25 \%$ and $37.5 \%$, respectively in ASAP group. Significant increase in PCa rate was reported on second biopsy in ASAP group when compares to HGPIN group $(37.5 \%$ vs $20.6 \%, \mathrm{p}=0.04)$ and BPT group $(37.5 \%$ vs $18.8 \%, \mathrm{p}=0.009)$. Overall, PCa was diagnosed in $26.5 \%, 45.8 \%, 18.8 \%$ in HGPIN, ASAP, BPT groups, respectively. Significant difference in PCa rate was detected only in ASAP group. PSAD has significant effects on $\mathrm{PCa}$ in all groups $(\mathrm{p}=0.001$ and $\mathrm{p}=0.01$, respectively). HGPIN is no longer associated with higher risk of cancer. Patients should be followed with yearly prostate specific antigen (PSA) and digital rectal examination (DRE). Repeat biopsy should be made as soon as feasible in patients with ASAP.

J. Exp.Clin.Med., 2013; 30: 241-245

(C) 2013 OMU

\section{Introduction}

Atypical small acinar proliferation (ASAP) and high grade prostatic intraepithelial neoplasia (HGPIN) are two common findings observed on prostate biopsies. In 5 to $10 \%$ of biopsy cases does not reveal clearly benign or malignant findings but rather HGPIN and/or ASAP which are considered to be predictors of subsequent prostatic adenocarcinoma (Epstein and Herawi, 2006). Prostatic intraepithelial neoplasia (PIN) was first described by Bostwick and Brawer in 1987 and defines architecturally benign prostatic acini and ducts lined by atypical cells. Although these atypical cells share morphological, histochemical, immunohistochemical and genetic changes with cancer, PIN lacks invasion of the basement membrane of the prostatic glands. PIN is usually multifocal and can involve clusters of glandular structures.
The incidence of HGPIN shows marked variation in the literature ranging from $0 \%$ to $24.6 \%$; the mean reported incidence is $7.7 \%$ (median 5.2\%) on needle biopsies with a $23 \%$ to $79 \%$ risk of carcinoma on subsequent biopsy (Zlotta et al., 1996; Bishara et al., 2004). Bostwick et al. (1993) also described ASAP as denoting the presence of suspicious glands with insufficient cytological or architectural atypia for a definitive cancer diagnosis. An average of $5 \%$ of needle biopsy pathology reports shows a diagnosis of atypical glands suspicious for carcinoma (Epstein and Herawi, 2006). The clinical importance of ASAP is its high predictive value for Prostate carcinoma (PCa) in subsequent biopsies. It has been reported that the cancer detection rate varies between $21 \%$ and $51 \%$ on the second biopsy in patients with ASAP (Borboroglu et al., 2000; Leite et al., 2008). 
Prostatic repeat biopsy is usually recommended following diagnosis of HGPIN or ASAP, although number of procedures remains still unclear. Knowing the rate of $\mathrm{PCa}$ diagnosis on repeat biopsies would aid primary treating physicians in the decision-making process for suspicious cases. For this purpose, we reviewed the outcomes of patients undergoing repeat biopsies, following initial diagnosis of HGPIN or ASAP and compared the pathological results to second biopsies due to increased prostate specific antigen (PSA).

\section{Material and methods}

We retrospectively assessed 1451 transrectal ultrasound guided prostate biopsy (TRUSBP) performed at our institution between January 2003 and March 2011, using an extended peripheral zone biopsy scheme. Indications for TRUSBP were: Abnormal digital rectal examination and/or a serum PSA over $2.5 \mathrm{ng} / \mathrm{ml}$. This scheme was defined by 10 to 22 (mean 16.3) peripheral zone biopsy cores incorporating the lateral horn with transition zone biopsy.

None of these patients had previous prostate biopsies. All patients were thoroughly examined by transrectal ultrasound (TRUS) before biopsy and prostate volumes were calculated by using an ellipsoid prostate formula. All of the lesions detected by TRUS were noted. Local anesthesia of $5 \mathrm{cc}$ lidocaine $(1 \%)$ was applied to prostate apex and base. All of the patients were placed in the left lateral decubitus position and all were examined with no bowel preparation. We used an 18 $\mathrm{G}$ core biopsy needle mounted on a spring-loaded automatic biopsy gun. All of the patients underwent a 10-core biopsy protocol with additional one core from each suspicious area detected by TRUS. In repeat biopsies, additional transitional zone sampling was added on both lobes. All patients underwent the same biopsy protocol regardless of the prostate gland size. All of the patients tolerated the biopsy procedure well and none of the patients needed intravenous sedation or narcotic analgesics. All taken biopsy specimens were labeled according to their localizations on prostate from where the biopsy was obtained and submitted separately in $10 \%$ formalin-filled containers to the Department of Pathology of our University Hospital.

We retrospectively reviewed our database to obtain PSA level and PSA density (PSAD) at the time of biopsy, prostatic volume, patient age, digital rectal examination (DRE) or TRUS results, time to the repeat biopsy, and the location of the ASAP, HGPIN and PCa according to needle biopsy results. When patients with an initial diagnosis of HGPIN or ASAP underwent another biopsy, four diagnoses were made: benign tissue, ASAP, HGPIN, or PCa. When performing analyses that compared the group with cancer with that with no cancer, we combined benign tissue and ASAP or HGPIN in a no-cancer group.

Clinical data were analyzed with Chi-square tests and Kruskal-Wallis test; in addition to these, binary logistic regression was performed to identify any correlation between the rates of cancer detection on repeat biopsy with the previously mentioned variables, while controlling for potentially confounding factors.

\section{Results}

Among the 1451 prostate biopsies taken 441 (30.4\%) were diagnosed as PCa, $93(6.4 \%)$ as HGPIN and 68 (4.7\%) as
ASAP. Among patients with HGPIN and ASAP, 68 patients and 48 patients were selected with subsequent biopsies due to diagnosis of HGPIN and ASAP, respectively.

We also selected 128 patients with the diagnosis of benign prostatic tissue (BPT) after first biopsy and we performed the repeat biopsies due to increased PSA values to compare the results with HGPIN and ASAP groups. Patient characteristics according to the pathology report on first biopsy are showed as a table (Table 1).

\begin{tabular}{lllll}
\multicolumn{4}{c}{$\begin{array}{c}\text { Table 1. Patient characteristics } \\
\text { report on first biopsy }\end{array}$} & \multicolumn{3}{l}{ according to the pathology } \\
& HGPIN & ASAP & BPT & $p$ \\
\hline Number & 68 & 48 & 128 & \\
Age & $65.5 \pm 1.9$ & $62.6 \pm 2.2$ & $62 \pm 1.2$ & 0.06 \\
PSA (ng/ml) & $10.3 \pm 1.3$ & $9.5 \pm 1.1$ & $10.6 \pm 0.8$ & 0.45 \\
DRE positive (\%) & 23.5 & 33.3 & 34.1 & 0.30 \\
TRUS positive (\%) & 20.8 & 29.4 & 26 & 0.58 \\
Prostate vol (cc) & $45 \pm 5$ & $41.3 \pm 4.8$ & $42.7 \pm 3.3$ & 0.88 \\
PSAD (ng/ml/cc) & $0.26 \pm 0.04$ & $0.27 \pm 0.04$ & $0.3 \pm 0.03$ & 0.42 \\
TZ-PSAD (ng/ml/cc) & $0.64 \pm 0.01$ & $0.55 \pm 0.08$ & $0.72 \pm 0.14$ & 0.51 \\
F/T PSA (\%) & $20.3 \pm 5$ & $17 \pm 3.6$ & $16.2 \pm 2$ & 0.62 \\
Mean repeat biopsies & $1.36 \pm 0.18$ & $1.37 \pm 0.12$ & $1.19 \pm 0.09$ & $0.025^{*}$ \\
Weeks between biopsies & $17.7 \pm 4$ & $14.6 \pm 3.8$ & $21 \pm 4$ & $0.028^{*}$ \\
\hline
\end{tabular}

Chi-square test and Kruskal-Wallis test were used for statistical analysis. *Significance between Benign and the other groups. There was no difference between HGPIN and ASAP groups.

HGPIN: High grade prostatic intraepithelial neoplasia; ASAP: Atypical small acinar proliferation; BPT: Benign prostatic tissue; PSA: Prostate specific antigen; DRE: Digital rectal examination; TRUS: Transrectal ultrasound; PSAD-TZ: Transitional zone PSA density.

In the HGPIN group, 68 patients underwent a second biopsy, whereas, 29 (42.6\%), and $3(4.4 \%)$ patients also underwent third, and fourth biopsies, respectively. Similarly, in the ASAP group, all 48 patients underwent a second biopsy, whereas $14(29.2 \%)$, and $4(8.3 \%)$ patients also underwent third, and fourth biopsies, respectively. In the BPT group, all 128 patients underwent a second biopsy, whereas $19(14.8 \%)$ and $6(4.6 \%)$ patients underwent the third and fourth biopsies, respectively. The distribution of the pathological results of patients who underwent repeat biopsies according to previous biopsies are showed as a table (Table 2).

After the second biopsy, diagnosis of HGPIN was confirmed in HGPIN group in 20 of 68 patients $(29.4 \%)$, whereas BPT and $\mathrm{PCa}$ were reported in 31 of 68 patients

\begin{tabular}{|c|c|c|c|c|}
\hline & HGPIN & ASAP & BPT & $p$ \\
\hline $\begin{array}{l}\text { Cancer after } 2 \text { nd biopsy } \\
(\%)\end{array}$ & $\begin{array}{l}14 / 68 \\
(20.6)\end{array}$ & $\begin{array}{l}18 / 48 \\
(37.5)\end{array}$ & $\begin{array}{l}24 / 128 \\
(18.8)\end{array}$ & $0.027 *$ \\
\hline $\begin{array}{l}\text { Overall cancer rate after } \\
\text { rebiopsies }(\%)\end{array}$ & $\begin{array}{l}18 / 68 \\
(26.5)\end{array}$ & $\begin{array}{l}22 / 48 \\
(45.8)\end{array}$ & $\begin{array}{l}24 / 128 \\
(18.8)\end{array}$ & $0.01 *$ \\
\hline Tumor foci & $2.83 \pm 0.56$ & $2.63 \pm 0.7$ & $3.2 \pm 1.1$ & 0.66 \\
\hline Gleason score $\geq 7$ & $\begin{array}{l}2 / 18 \\
(11.1 \%)\end{array}$ & $\begin{array}{l}3 / 22 \\
(13.6 \%)\end{array}$ & $\begin{array}{l}6 / 24 \\
(25 \%)\end{array}$ & 0.43 \\
\hline $\begin{array}{l}\text { Cancer with single focus } \\
\text { HGPIN }(\%)\end{array}$ & & \multicolumn{2}{|c|}{$3 / 27(11.1)$} & 0.01 \\
\hline $\begin{array}{l}\text { Cancer with HGPIN } \\
\text { multifoci }(\%)\end{array}$ & & \multicolumn{2}{|c|}{$15 / 41(36.6)$} & \\
\hline
\end{tabular}

*There was no difference between HGPIN and Benign group in terms of cancer detection rate.

HGPIN: High grade prostatic intraepithelial neoplasia; ASAP: Atypical small acinar proliferation; BPT: Benign prostatic tissue. 


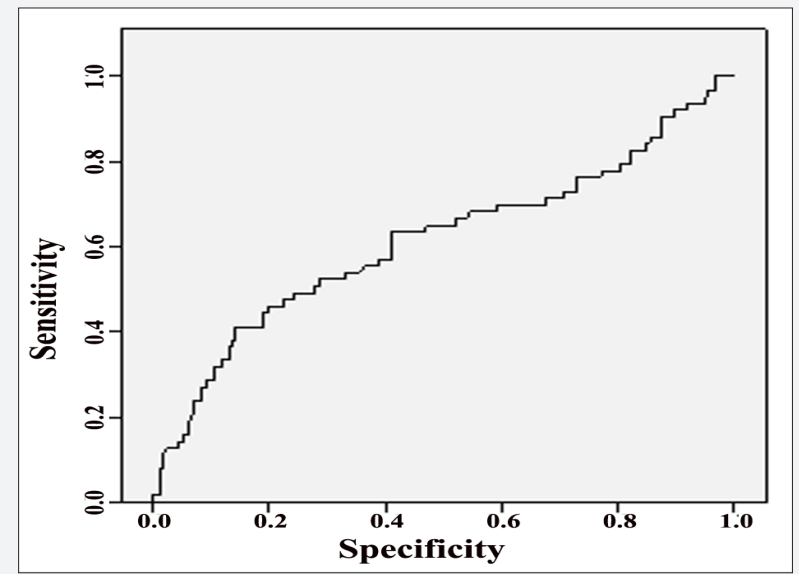

Fig. 1. Receiver operating characteristic (ROC) curve of PSAD in all patients for detection of prostate cancer. (Area under curve is $0.615, \mathrm{p}=0.007$ )

(45.6\%) and in 14 of 68 patients (20.6\%), respectively; in ASAP group, the diagnosis of ASAP was confirmed in 12 of 48 patients $(25 \%)$, whereas the diagnosis of BPT and PCa were the same rate of $(37.5 \%)$ in 18 patients. In the BPT group, PCa was detected in 24 of 128 patients (18.8\%) after second biopsy. Statistically significant differences regarding $\mathrm{PCa}$ detection rates were not detected at the second biopsies between HGPIN and BPT groups (20.6\% vs $18.8 \%$ respectively, $\mathrm{p}=0.757)$. However a significant increase in $\mathrm{PCa}$ rate was reported at the second biopsy in ASAP group when compares to HGPIN group $(37.5 \%$ vs $20.6 \%, \mathrm{p}=0.04)$ and BPT group (37.5\% vs $18.8 \%, \mathrm{p}=0.009)$.

Overall, $\mathrm{PCa}$ was diagnosed in 18 of 68 patients $(26.5 \%)$ in HGPIN, in 22 of 48 patients (45.8\%) in ASAP group, in 24 of 128 patients $(18.8 \%)$ in BPT group. No statistical significance in terms of $\mathrm{PCa}$ detection rates after repeat biopsies between HGPIN and BPT groups $(26.5 \%$ vs $18.8 \%$ respectively, $\mathrm{p}=0.21$ ) was observed. However a significant increase in overall PCa rate was reported after repeat biopsies in ASAP group when compares to HGPIN group $(45.8 \%$ vs $26.5 \%$, $\mathrm{p}=0.03$ ) and BPT group (45.8\% vs $18.8 \%, \mathrm{p}=0.001)$.

Pathologic evaluation of the 18 patients with PCa previously diagnosed as HGPIN showed a Gleason sum greater than 7 in 2 cases $(11.1 \%$ ), among the 22 patients in ASAP group with $\mathrm{PCa}$, a Gleason sum greater than 7 in 3 cases (13.6\%), whereas among 24 patients in BPT group with $\mathrm{PCa}$, a Gleason sum greater than 7 in 6 cases $(25 \%)$. However, no significant difference was detected in the distribution of PCa grading in the three groups of patients (Table 2).

Patients were divided in 2 groups according to the number of cores involved with HGPIN on initial biopsy. There were 3 patients $(11.1 \%$ ) with PCa among 27 patients with 1 core involved with HGPIN. On the other hand, there were 15 patients $(36.6 \%)$ with $\mathrm{PCa}$ among 41 patients with 2 cores or more. Difference was statistically significant between these 2 groups $(\mathrm{p}=0.01)$.

Considering all patients, the results of binary logistic regression analysis showed that age of patient, PSA levels, DRE or TRUS findings were not significant predictors of PCa. Only PSAD has significant effects for the detection of prostate cancer in all groups $(\mathrm{p}=0.001)$. When considering the HGPIN and ASAP groups separately, the only factor predictive of malignancy was PSAD in the binary logistic regression analysis $(\mathrm{p}=0.01, \mathrm{p}=0.04$ respectively). Receiver operating characteristics curves for PSAD was shown in the (Fig. 1). The area under the curve for PSAD was $61.5 \%$ $(\mathrm{p}=0.007)$.

Considering that our PCa detection rate was $30.6 \%$ after first biopsy, in those patients with HGPIN and ASAP, second, third and fourth biopsy added further $2.2 \%, 0.4 \%$ and $0.1 \%$ $\mathrm{PCa}$ detection rate respectively.

\section{Discussion}

Significant advances have been achieved in the diagnosis of PCa with the introduction of PSA and prostate biopsy techniques. However, the histological diagnosis of PCa in biopsy specimens remains a challenge for pathologists. In the prostate biopsies performed for suspected $\mathrm{PCa}$, positive results confirm the diagnosis; however, the suspicion of cancer remains with negative results. Moreover, the suspicion of cancer becomes more prominent if the results of the biopsy reveal HGPIN or ASAP (Koca et al., 2011).

HGPIN was considered as precursor of $\mathrm{PCa}$ and a predictor of PCa on subsequent biopsies, especially in the era of sextant biopsy and repeat biopsy has become a standard of care for HGPIN for almost a decade (Kronz et al., 2001 Moore et al., 2005) The incidence of HGPIN shows marked variation in the literature ranging from $0 \%$ to $24.6 \%$; the mean reported incidence is $7.7 \%$ (median 5.2\%) (Epstein and Herawi, 2006). In a study with a large, community based prostate biopsy population, the incidence of HGPIN was reported as 3.0\% in 42,667 patients (Girasole et al., 2006). In our series, the incidence was $6.4 \%$ and within the reported range. The false-negative rates of the sextant biopsy scheme became apparent after the standard practice has shifted to a more extensive sampling of the peripheral zone (Roehrborn et al., 1996; Fleshner et al., 1997). This shift has resulted in a nearly $30 \%$ increase in the cancer detection rate at initial biopsy (Babaian et al., 2000; Presti et al., 2000). However, the cancer detection rate on repeat biopsies in patients previously diagnosed with HGPIN is progressively decreasing with the increasing use of extended biopsy techniques (Eskew et al., 1997; Presti et al., 2000; Herawi et al., 2006). In a review by Epstein et al. the risk of cancer after a repeat biopsy for benign disease was 23\% versus $18.7 \%$ for HGPIN (Epstein and Herawi, 2006). In this current study, risk of cancer after repeat biopsies for benign and HGPIN was $18.8 \%$ versus $26.5 \%$, respectively. We did not observe significant differences between benign and HGPIN groups regarding PCa detection. Similarly, Naya et al. (2004) and Gallo et al. (2008) showed no statistical difference in the risk of cancer on repeat biopsy following a HGPIN diagnosis compared to repeat biopsy after a benign diagnosis. In addition, Lefkowitz et al (2002) suggested that early repeat biopsy was not mandatory within the one year but approximately a quarter of men with HGPIN will have clinically detectable PCa during 3 years follow-up. So they recommended that all men with HGPIN undergo biopsy 3 years after diagnosis regardless of change in serum PSA. However there was no group of patients with benign diagnosis after first biopsy to compare to HGPIN regarding PCa during 3 years follow up. Howover, repeat biopsy should be performed if HGPIN is detected by a sextant biopsy technique, and if the biopsy has been performed with multiple sampling, other parameters, such as PSA or rectal 
examination status, should be considered (Moore et al., 2005 Epstein and Herawi, 2006; Eskıcorapci et al., 2007; Orderda and Gontero, 2009). It is important to note that the detection of PCa on repeat biopsies in patients with HGPIN detected in their first biopsies is not a missed diagnosis in the initial biopsy, but in fact might reflect the development of a new cancer arising from HGPIN (Meng et al., 2003).

Increasing number of studies show that the number of cores with HGPIN can help predict which patients are at higher risk for cancer following a biopsy with the diagnosis of HGPIN (Merrimen et al., 2011). Roscigno et al. (2004) reported that cancer detection was significantly greater in patients with plurifocal HGPIN than in those with monofocal HGPIN (70\% vs. 10\%, respectively; P <0.005) (Roscigno et al., 2004). Similarly Netto and Epstein (2006), Merrimen et al., (2009) and De Nunzio et al. ( 2009)reported the increased incidence of prostatic carcinoma on second biopsy in patients with at least 4 cores involved with HGPIN defined as widespread HGPIN. In our study, we observed that cancer detection rate was significantly higher in patients with 2 cores or more involved with HGPIN than in those with monofocal HGPIN on second biopsy (36.6\% vs. $11.1 \%$, respectively; $\mathrm{P}=0.01)$.

Like HGPIN, ASAP is also a rare isolated finding on initial needle biopsy. The $4.7 \%$ incidence of ASAP on initial biopsy in our series is similar to the average rate of $5 \%$ reported by Epstein and Herawi (2006). The average risk of cancer following an atypical diagnosis is $40 \%$ on repeat biopsies so a repeat biopsy is recommended to all patients with ASAP.

Iczkowski et al. ( 1998 reported the 295 patients with ASAP, of whom $112(38 \%)$ were diagnosed cancer on repeat biopsy, 12 of the $36(33 \%)$ patients on second repeat biopsy and 1 of the $8(12.5 \%)$ patients on third repeat biopsy. Thus the nearly $85 \%$ of the cancer was diagnosed after first repeat biopsy. Similarly, Moore et al. (2005) examined the 53 patients with ASAP in whom $85 \%$ of cancer was diagnosed on first repeat biopsy. In our study, 18 of $22(84 \%)$ cancer were diagnosed on first repeat biopsy and 21 of $22(94.5 \%)$ cancer were diagnosed on second repeat biopsy in ASAP group.

Many investigators have attempted to determine clinical and pathological predictors of cancer risk in patients with ASAP and HGPIN. Some of them have noted no correlation between patient age, DRE, TRUS findings, serum PSA and cancer risk (Moore et al., 2005; Borboroglu et al., 2001; Abouassaly et al., 2008). On the other hand, some authors noted that age, PSAD, transitional zone PSA density (PSAD-TZ) and the number of cores with HGPIN were found to predict cancer in another series of HGPIN patients (Abdel-Khalek et al., 2004; Eskicorapci et al., 2007). In this study, we found that the only predictive factor for cancer was the PSAD in both ASAP and HGPIN groups.

Several limitations should be considered when evaluating the findings of this study. Data were collected retrospectively from a database at a single tertiary care institution. So our variables were obtained from clinical chart review they were inherently incomplete. Biopsy samples were not examined by the same pathologist, although all biopsies were examined in the same pathology laboratory. The number of patients with ASAP and HGPIN is relatively small, and this may increase the possibilty that our findings are due to chance.

In conclusion, ASAP and HGPIN entities have different histological findings and more importantly for patient care the finding of ASAP is associated with a much higher risk of cancer on repeat biopsy compared to HGPIN and benign groups. The finding of ASAP on prostate biopsy is associated with an approximately $50 \%$ likelihood of underlying malignancy. In this selected group, $94.5 \%$ of cancer was diagnosed on second repeat biopsy. Therefore, in these patients a recommendation of repeat biopsy should be made as soon as feasible. Further information is required for third repeat biopsy in this selected group.

\section{REFERENCES}

Abdel-Khalek, M., El-Baz M., Ibrahiem el, H., 2004. Predictors of prostate cancer on extended biopsy in patients with high-grade prostatic intraepithelial neoplasia: A multivariate analysis model. BJU Int. 94, 528-533.

Abouassaly, R., Tan, N., Moussa, A., Jones, J.S., Chen, M., 2008. Risk of prostate cancer after diagnosis of atypical glands suspicious for carcinoma on saturation and traditional biopsies. J. Urol. 180, 911-914.

Babaian, R.J., Toi, A., Kamoi, K., Troncoso, P., Sweet, J., Evans, R., Johnston, D., Chen, M., 2000. A comparative analysis of sextant and an extended 11-core multisite directed biopsy strategy. J. Urol, 163, 152-157.

Bishara, T., Ramnani, D., Epstein, J., 2004. Highgrade prostatic intraepithelial neoplasia on needle biopsy: Risk of cancer on repeat biopsy related to number of involved cores and morphologic pattern. Am. J. Surg. Pathol. 28, 629.

Borboroglu, P.G., Comer, S.W., Riffenburgh, R.H., Amling, C.L., 2000. Extensive repeat transrectal ultrasound guided prostate biopsy in patients with previous benign sextant biopsies. J. Urol. 163, 158-162.

Borboroglu, P., Sur, R., Roberts, J., Amling, C., 2001. Repeat biopsy strategy in patients with atypical small acinar proliferation or high grade prostatic intraepithelial neoplasia on initial prostate needle biopsy. J. Urol. 166, 866-870.

Bostwick, D., Brawer, M., 1987. Prostatic intraepithelial neoplasia and early invasion in prostate cancer. Cancer. 59, 788.

Bostwick, D., Srigley, J., Grignon, D., Maksem, J., Humphrey, P., van der Kwast, T.H., Bose, D., Harrison, J., Young, R.H., 1993. Atypical adenomatous hyperplasia of the prostate: Morphologic criteria for its distinction from well-differentiated carcinoma. Hum. Pathol. $24,819-832$.

De Nunzio, C., Trucchi, A., Miano, R, Stoppacciaro, A., Fattahi, H., Cicione, A., Tubaro, A., 2009. The number of cores positive for high grade prostatic intraepithelial neoplasia on initial biopsy is associated with prostate cancer on second biopsy. J. Urol. 181, 1069-1074.

Epstein, J.I., Herawi, M., 2006. Prostate needle biopsies containing prostatic intraepithelial neoplasia or atypical foci suspicious for carcinoma: Implications for patient care. J. Urol. 175, 820-834.

Eskew, L.A., Bare, R.L., McCullough, D.L., 1997. Systematic 5 region prostate biopsy is superior to sextant method for diagnosing carcinoma of the prostate. J. Urol. 157, 199-202.

Eskicorapci, S., Guliyev, F., Islamoglu, E., Ergen, A., Ozen, H., 2007. The effect of prior biopsy scheme on prostate cancer detection for repeat biopsy population: Results of the 14-core prostate biopsy technique. Int. Urol. Nephrol. 39, 189-195.

Fleshner, N.E., O’Sullivan, M., Fair, W.R., 1997. Prevalence and predictors of a positive repeat transrectal ultrasound guided needle biopsy of the prostate. J. Urol. 158, 505-508. 
Gallo, F., Chiono, L., Gastaldi, E., Venturino, E., Giberti, G., 2008. Prognostic significance of high-grade prostatic intraepithelial neoplasia (hgpin): Risk of prostatic cancer on repeat biopsies. Urology. 72, 628-32.

Girasole, C., Cookson, M., Putzi, M., Chang, S.S., Smith, J.A., Jr., Wells, N., Oppenheimer, J.R., Shappell, S.B., 2006. Significance of atypical and suspicious small acinar proliferations, and high grade prostatic intraepithelial neoplasia on prostate biopsy: Implications for cancer detection and biopsy strategy. J. Urol. 175, 929-933.

Herawi, M., Kahane, H., Cavallo, C., Epstein, J., 2006. Risk of prostate cancer on first re-biopsy within 1 year following a diagnosis of high grade prostatic intraepithelial neoplasia is related to the number of cores sampled. J. Urol. 175, 121-124.

Iczkowski K., Bassler T., Schwob V., Bassler, I.C., Kunnel, B.S., Orozco, R.E., Bostwick, D.G., 1998. Diagnosis of "suspicious for malignancy" in prostate biopsies: Predictive value for cancer. Urology. 51, 749-757.

Koca, O., Caliskan, S., Ozturk, M.I., Gunes, M., Karaman, M.I., 2011. Significance of atypical small acinar proliferation and high-grade prostatic intraepithelial neoplasia in prostate biopsy. Korean J. Urol. 52, 736-740.

Kronz, J.D., Shaikh, A.A., Epstein, J.I., 2001. High-grade prostatic intraepithelial neoplasia with adjacent small atypical glands on prostate biopsy. Hum. Pathol. 32, 389-395.

Lefkowitz, G,. Taneja, S., Brown, J., Melamed, J., Lepor, H., 2002. Followup interval prostate biopsy 3 years after diagnosis of high grade prostatic intraepithelial neoplasia is associated with high likelihood of prostate cancer, independent of change in prostate specific antigen levels. J. Urol. 168, 1415-1418.

Leite, K.R., Srougi, M., Dall'Oglio, M.F., Sanudo, A., Camara-Lopes, L.H., 2008. Histopathological findings in extended prostate biopsy with PSA < or $=4$ ng/mL. Int. Braz. J. Urol. 34, 283-290.

Meng, M.V., Shinohara, K., Grossfeld, G.D., 2003. Significance of high-grade prostatic intraepithelial neoplasia on prostate biopsy. Urol. Oncol. 21, 145-151.

Merrimen, J.L., Jones, G., Walker, D., Leung, C.S., Kapusta, L.R., Srigley, J.R., 2009. Multifocal high grade prostatic intraepithelial neoplasia is a significant risk factor for prostatic adenocarcinoma. J. Urol. 182, 485-490.

Merrimen, J.L., Jones, G., Hussein, S.A., Leung, C.S., Kapusta, L.R., Srigley, J.R., 2011 . A model to predict prostate cancer after atypical findings in initial prostate needle biopsy. J. Urol. 185, 1240-1245.

Moore, C.K., Karikehalli, S., Nazeer, T., Fisher, H.A., Kaufman, R.P., Jr., Mian, B.M., 2005. Prognostic significance of high grade prostatic intraepithelial neoplasia and atypical small acinar proliferation in the contemporary era. J. Urol. 173, 70-72.

Naya, Y., Ayala, A., Tamboli, P., Babaian, R., 2004. Can the number of cores with high-grade prostate intraepithelial neoplasia predict cancer in men who undergo repeat biopsy? Urology. 63, 503-508.

Netto, G., Epstein, J., 2006. Widespread high-grade prostatic intraepithelial neoplasia on prostatic needle biopsy: A significant likelihood of subsequently diagnosed adenocarcinoma. Am. J. Surg. Pathol. 30, 1184-1188.

Oderda, M., Gontero, P., 2009. High-grade prostatic intraepithelial neoplasia and atypical small acinar proliferation: Is repeat biopsy still necessary? BJU Int. 104, 1554-1556.

Presti, J.C., Jr., Chang, J.J., Bhargava, V., Shinohara, K., 2000. The optimal systematic prostate biopsy scheme should include 8 rather than 6 biopsies: Results of a prospective clinical trial. J .Urol. 163, 163-166.

Roehrborn, C.G., Pickens, G.J., Sanders, J.S., 1996. Diagnostic yield of repeated transrectal ultrasound-guided biopsies stratified by specific histopathologic diagnoses and prostate specific antigen levels. Urology. 47, 347-352.

Roscigno, M., Scattoni, V., Freschi, M., Raber, M., Colombo, R., Bertini, R., Montorsi, F., Rigatti, P., 2004. Monofocal and plurifocal high-grade prostatic intraepithelial neoplasia on extended prostate biopsies: Factors predicting cancer detection on extended repeat biopsy. Urology. 63, $1105-1110$.

Zlotta, A.R., Raviv, G., Schulman, C.C., 1996. Clinical prognostic criteria for later diagnosis of prostate carcinoma in patients with initial isolated prostatic intraepithelial neoplasia. Eur. Urol. 30, 249-255. 